\title{
Assessment of Genetic Variability of a Set of Maize Inbred Lines for Drought Tolerance under Temperate Conditions
}

\author{
Asima Gazal $^{1 *}$, F.A. Nehvi², Ajaz Ahmad Lone ${ }^{3}$ and Zahoor Ahmed Dar ${ }^{3}$ \\ ${ }^{1}$ Division of Genetics and Plant Breeding, SKUAST-K, J\&K, India \\ ${ }^{2}$ Saffron Research Station, Pampore, SKUAST-K, J\&K, India \\ ${ }^{3}$ Dryland Agricultural Research Station, SKUAST-K, Budgam, J\&K, India \\ *Corresponding author
}

\begin{tabular}{|l|}
\hline Ke y w o r d s \\
Drought, Maize, \\
Relative water \\
content, Anthesis- \\
silking interval, \\
Chlorophyll \\
content.
\end{tabular}

\section{A B S T R A C T}

Lack of hybrids/composites showing tolerant genes for moisture stress is responsible for low maize productivity in Kashmir. Present study was an attempt in this direction to assess the genetic variability of 100 homozygous maize inbred lines at different stages of selfing during kharif 2013 and 2014 at AICRP (Maize)-Srinagar Centre. Inbred lines were evaluated for obtaining information on genetic variability, heritability and genetic advance for maturity, morphological, physiological, yield and quality traits. A wide range of variability revealing significant response of main effects (lines, irrigations and years and their respective digenic and trigenic interactions) was observed. Upper range values suggest presence of elite lines exhibiting taller plants associated with more number of ears plant $^{-1}$, more ear height, more number of kernels row ${ }^{-1}$, heavier grains, high yielders, better quality, high leaf relative water content, more chlorophyll content, earliness towards tasseling, silking, anthesis-silking interval, maturity and low canopy temperatures associated with low stomatal count. Medium to high values of genetic coefficient of variability accompanied with high heritability and high genetic gain (as \% of mean) were exhibited for anthesis-silking interval, leaf relative water content, stomatal count, chlorophyll content before flowering, chlorophyll content before maturity, ears plant ${ }^{-1}$, grain yield plot $^{-1}$, protein content all confirming that these traits can be given more weightage while applying selection for improvement of these traits and in identifying elite drought tolerant lines.

\section{Introduction}

Maize is one of the most important cereal crops globally after wheat and rice and holds a unique position in world agriculture as food, feed and source of diverse industrially important products. The most important abiotic stress affecting maize crop production worldwide is drought, defined as the mechanism causing minimum loss of yield in a water deficit environment relative to the maximum yield in a water constraint free management of the crop. It is becoming one of the topmost constraints affecting the production and productivity of maize worldwide (Araus et al., 2002). One of the major limiting factors for maize production and productivity is inadequate soil moisture particularly during flowering and grain filling stages (Joshi et al., 2005). Thus, breeding for maize drought tolerance is primarily aimed at identifying lines with optimal reproductive 
capacity with low yield penalty under drought-stress conditions as compared to well-watered conditions; this is further evaluated by assessing the stability of promising genotypes by conducting multiseason drought trials (Bolanos and Edmeades, 1993).

Maize is cultivated on an area of 0.31 million ha in $\mathrm{J} \& \mathrm{~K}$ with total production of 0.50 million tonnes and productivity of 1.6 tonnes ha $^{-1}$, whereas, 0.1 million ha area is under maize in Kashmir with production of 0.15 million tonnes with an average yield of 1.2 tonnes $\mathrm{ha}^{-1}$ (Anonymous, 2014). Out of 0.1 million ha area, under maize in Kashmir only 12 thousand ha is irrigated and the remaining 82 per cent is rainfed (DE and S, 2013). Maize requires $500-800 \mathrm{~mm}$ of water during life cycle of 80 to 110 days at critical stages of crop growth viz., knee height stage, flowering stage (tasseling and silking) and grain filling stage (Critchley and Klaus, 1991). But under valley conditions maize is deficit of water as only 307 to 555 (mm) of precipitation is received during the critical stages of crop growth (Anonymous, 2013). Therefore, lack of potential hybrids showing genes for drought tolerance is responsible for low maize productivity in Kashmir as compared to state average yield of 1.6 tonnes ha $^{-1}$. AICRP (Maize)-Srinagar Centre under All India Maize Improvement Project started genetic amelioration programme way back in 2002 and series of inbred lines were derived from the heterotic pools and also obtained from Directorate of Maize Research, New Delhi. Therefore, an attempt was made in this direction to identify inbred lines for inbuilt drought tolerance. The, effective identification of potentially useful and stress resilient germplasm forms the first and foremost step in a crop improvement programme. High yielding genotypes with drought tolerance could be directly utilized for undertaking a hybridization programme.
Yield is an ultimate criterion which a plant breeder has always to keep in view in his attempt to evolve improved cultivars of any crop. The genetic parameters like genotypic coefficient of variation, heritability and genetic advance are the tools in the hands of a plant breeder for ensuring efficient selection (Johnson et al., 1955). The present study therefore, is aimed at evaluating a set of homozygous inbred lines over years to identify potential drought tolerant line and to assess the utility of specific traits in breeding for production of drought tolerant single cross hybrids.

\section{Materials and Methods}

The genetic material selected for phenotyping under drought stress included a set of 100 inbred lines at different stage of selfing. The lines were evaluated in factorial RBD with two replications over two years in two row experimental plot of 1 meter length (spacing $60 \times 20 \mathrm{~cm}$ ) with recommended package of practices. The drought trials were carried out at AICRP (Maize)-Srinagar Centre, SKUAST-K during Kharif 2013 and 2014 each having four different water treatments/environments: (i) Well Watered, (ii) Intermediate Stress, (iii) Mild Stress and (iv) Stress viz.,

Well Watered (WW): Irrigated at knee height stage, flowering and grain filling stages.

Intermediate Stress (IS): Irrigated at knee height stage and flowering stage.

Mild Stress (MS): Irrigated at knee height stage and

Stress (S): Rainfed.

The meteorological data, including minimum and maximum temperatures, relative humidity (RH) and rainfall were collected throughout 
the experimental period for both the years (Banziger et al., 2000). The traits recorded were : days to $50 \%$ tasseling, days to $50 \%$ silking, anthesis-silking interval, days to maturity, plant height $(\mathrm{cm})$, ear height $(\mathrm{cm})$, leaf relative water content (\%), canopy temperature before flowering $\left({ }^{\circ} \mathrm{C}\right)$, canopy temperature before maturity $\left({ }^{\circ} \mathrm{C}\right)$, stomatal count $\left(\mathrm{mm}^{-2}\right)$, chlorophyll content before flowering (SPAD units), chlorophyll content before maturity (SPAD units), ears plant $^{-1}$ (EPP), kernels row ${ }^{-1}, 100$ grain weight $(\mathrm{g})$, grain yield plot $^{-1}(\mathrm{~g})$ and protein content $(\%)$.

The number of ears per plant was measured on the basis of total number of ears harvested in each plant. For grain yield per plot, the ears of five plants per replication per treatment in each plot were shelled and weighed. Moisture content of each progeny was determined by moisture meter from a representative sample of shelled grains. The grain yield was later adjusted to 15 per cent moisture and mean grain yield plot $^{-1}(\mathrm{~g})$ calculated. Leaf relative water content was estimated as per Barrs and Weatherly (1962) with formula:

$\operatorname{RWC}(\%)=[(\mathrm{Fw}-\mathrm{Dw}) /(\mathrm{Tw}-\mathrm{Dw})] \times 100$.

Where, Fw, Dw, and Tw stands for fresh weight, dry weight and turgid weight, respectively. Canopy temperature was measured by infra-red thermometer (Fluke 62 MAX - FLUKE Corporation, USA) by recording temperature at 13.00-15.00 from fully exposed leaves to sun light. Chlorophyll content was measured by Chlorophyll metre SPAD meter (Hanstech, Model CL-01) before flowering and before maturity and was expressed in SPAD value. Protein content was measured by NIR (Model CROPSCAN 2000G/2000B) at Seed Processing Unit SKUAST-K, J\&K, India. Pooled analysis of variance (ANOVA) was used to quantify the genetic differences among the genotypes. Data thus generated was analyzed using
WINDOSTAT version 9.2 software for various statistical parameters.

\section{Results and Discussion}

Analysis of variance revealed highly significant mean sum of squares for the maize inbred lines under study for all the traits thus indicating significant difference amongst the maize lines. Mean sum of squares due to years and irrigations were also significant for all the traits indicating differential responses of maize lines for these traits over years and different moisture management regimes (Tables 1, 2, 3). Replication mean sum of squares exhibited significant differences among replications for all the traits except days to $50 \%$ silking, days to $50 \%$ tasseling, anthesis-silking interval, days to maturity, canopy temperature before flowering, canopy temperature before maturity, grain yield plot $^{-1}$. Two way interactions viz., line $\mathrm{x}$ year, line $\mathrm{x}$ irrigation and irrigation $\mathrm{x}$ year exhibited highly significant mean sum of squares for all the traits except line $\mathrm{x}$ year for leaf relative water content, stomatal count, chlorophyll content before flowering, chlorophyll content before maturity and line $\mathrm{x}$ irrigation for canopy temperature before flowering. Three way interactions (lines $\mathrm{x}$ irrigation $\mathrm{x}$ year) were observed to be significant for all the traits except for days to $50 \%$ tasseling, leaf relative water content, canopy temperature before flowering, canopy temperature before maturity, stomatal count, chlorophyll content before flowering, chlorophyll content before maturity, ears plant $^{-1}$, kernels row ${ }^{-1}, 100$ grain weight and protein content. Similarly, Dubey et al., (2010) reported presence of significant genetic variation for all the drought related traits studied under different moisture management regimes revealing importance of locations/seasons, environments, location/ season $\times$ treatment and environment $\mathrm{x}$ treatment interaction for almost all the characters. Results were in conformity with 
Chapman et al., (1997); Banziger et al., (2000); Zaidi et al., (2004), Saindass et al., (2001); Meseka et al., (2008), Nepolean et al., (2012); Azam et al., (2014); Abuali et al., (2014) and Umar et al., (2015). Estimates of components of variability for various traits revealed a wide range of variability for all the characters under study in Y1, Y2 and pooled analysis indicating ample scope for selection of elite maize lines exhibiting tolerance to moisture stress.

Perusal of Table 4 revealed that the range of variability was almost at par in both the years for all the maturity, morphological, yield, physiological and quality traits. However, for days to $50 \%$ tasseling, days to $50 \%$ silking, kernels row $^{-1}, 100$ grain weight, grain yield plot $^{-1}$, protein content, leaf relative water content, canopy temperature before flowering, canopy temperature before maturity, chlorophyll content before flowering, chlorophyll content before maturity superior population means were recorded in $\mathrm{Y} 1$ and plant height, ear height, ear plant ${ }^{-1}$ and stomatal count recorded superior population means in Y2. No differences in population means were observed for anthesis-silking interval and days to maturity in Y1 and Y2.

The magnitude of improvement of any quantitative trait primarily depends upon the realistic estimate of variability. It is therefore, important to assess the relative magnitude of components of variability in order to use such information together with other selection parameters for improvement of plant type through adoption of effective breeding methods (Johnson et al., 1955; Hanson et al., 1956; Williams, 1964; Briggs and Knowels, 1967). Components of phenotypic variability were higher than the corresponding estimates of genotypic variability for all the traits under study in individual years and pooled over years analysis, thereby revealing the importance of environmental variance in the expression of the traits. Kuchanur (2010); Salman et al., (2011) and Kumar et al., (2014) were observed similar results.

Genotypic coefficient of variation was high (> 20) for chlorophyll content before maturity, ears plant $^{-1}$ thus indicating presence of sufficient inherent genetic variance over which selection could be effective (Table 5). However, moderate values of GCV (10-20) were recorded for ASI, leaf relative water content, stomatal count, chlorophyll content before flowering, kernels row $^{-1}, 100$ grain weight, grain yield plot $^{-1}$ and protein content.

Similar results of moderate GCV were observed by Choudhary and Choudhary (2002); Alake et al., (2008); Salman et al., (2011); Praveenkumar and Sridevi (2014); and Kumar et al., (2014). High to moderate GCV for these traits indicated sufficient variability and offers scope to improve these traits through phenotypic selection. Maturity traits, plant height, ear height and canopy temperature showed low GCV estimates $(<10)$ therefore, there is a limited scope of selection. Confounding influence of line $\mathrm{x}$ year interaction of the genotypic variance resulted in considerable reduction of estimates in pooled analysis.

Therefore, selection on the basis of single year data is not advisable. GCV of populations grown under different moisture management regimes exhibited similarity in nomenclature (low, moderate and high) for all the traits except for ASI under stress conditions, ears plant ${ }^{-1}$ under well watered conditions and 100 grain weight for stress, intermediate stress and mild stress. Populations under study confirmed variability in performance for traits due to more influence of genotype rather than environment. This study was supported by Banziger et al., (2000) and Zaidi and Singh (2005). 
Table.1 Analysis of variance for maturity traits in inbred lines of maize (Zea mays L.) - Mean Sum of Squares (Pooled over years)

\begin{tabular}{|c|c|c|c|c|c|c|c|}
\hline Source of variation & d.f & DT & DS & ASI & DM & $\mathbf{P H}$ & EH \\
\hline Replications & 1 & 0.89 & 0.76 & 0.04 & 1.75 & $19214.12 * *$ & $4729.79 * *$ \\
\hline Year & 1 & $162.56 * *$ & $635.04 * *$ & $155.00 * *$ & $564.53 * *$ & $282454.50 * *$ & $72550.50 * *$ \\
\hline Irrigations & 3 & $332.15 * *$ & $718.91 * *$ & $78.54 * *$ & $1120.22 * *$ & $948795.20 * *$ & $243009.90 * *$ \\
\hline Lines & 99 & $285.58 * *$ & $380.82 * *$ & $8.86 * *$ & $452.36^{* *}$ & $1132.90 * *$ & $299.11 * *$ \\
\hline Lines $\times$ year & 99 & $0.42 * *$ & $2.13 * *$ & $1.06^{* * *}$ & $5.49^{* * *}$ & $8.54 * *$ & $6.13 * *$ \\
\hline Line $\times$ irrigation & 297 & $1.90 * *$ & $4.55^{* *}$ & $0.51 * *$ & $7.03 * *$ & $19.09 * *$ & $6.27 * *$ \\
\hline Irrigations $\times$ year & 3 & $6.84 * *$ & $8.21 * *$ & $0.18 *$ & $83.96 * *$ & $21026.50 * *$ & $5142.38 * *$ \\
\hline $\begin{array}{l}\text { Irrigation within replication } \\
\text { (Pooled over year) }\end{array}$ & 7 & 1.10 & 1.12 & 0.04 & 0.80 & $59.35 * *$ & $16.61 * *$ \\
\hline $\begin{array}{lcc}\text { Irrigation within years } & \text { within } \\
\text { replication (Pooled over year) } & \end{array}$ & 15 & $79.94 * *$ & $188.99 * *$ & $26.08 * *$ & $278.96 * *$ & $214103.26^{* *}$ & $54790.22 * *$ \\
\hline Lines $\times$ irrigation $\times$ year & 297 & 0.12 & $0.38 * *$ & $0.26 * *$ & $4.41 * *$ & 0.32 & 0.08 \\
\hline Error (Pooled over year) & 1485 & 0.99 & 1.40 & 0.26 & 5.06 & 6.91 & 3.08 \\
\hline
\end{tabular}

*, ** Significant at 5 and $1 \%$ level, respectively. Days to $50 \%$ tasseling = DT; Days to $50 \%$ silking = DS; Anthesis-silking interval = ASI; Days to maturity = DM; Plant height $(\mathrm{cm})=\mathrm{PH}$; Ear height $(\mathrm{cm})=\mathrm{EH}$

Table.2 Analysis of variance for physiological traits in inbred lines of maize (Zea mays L.) - Mean Sum of Squares (Pooled over years)

\begin{tabular}{|c|c|c|c|c|c|c|c|}
\hline Source of variation & d.f & LRWC & SC & CTF & CTM & $\mathbf{C C F}$ & CCM \\
\hline Replications & 1 & $1774.84 * *$ & $547.04 * *$ & 0.34 & 0.12 & $412.44 * *$ & $482.39 * *$ \\
\hline Year & 1 & $44426.25 * *$ & $4729.93 * *$ & $1132.10^{* *}$ & $873.03 * *$ & $802.26 * *$ & $120.13 * *$ \\
\hline Irrigations & 3 & $805287.00 * *$ & $2625.15 * *$ & $3738.55 * *$ & $2812.23 * *$ & $4460.39 * *$ & $732.05 * *$ \\
\hline Lines & 99 & $2194.18 * *$ & $1288.39 * *$ & $22.75 * *$ & $22.87 * *$ & $596.43 * *$ & $139.40 * *$ \\
\hline Lines $\times$ year & 99 & 4.39 & 0.48 & 0.03 & 0.10 & 0.09 & 0.04 \\
\hline Line $\times$ irrigation & 297 & 118.78 & 0.17 & $1.22 * *$ & $1.28 * *$ & 0.35 & 0.12 \\
\hline Irrigations $\times$ year & 3 & $10542.30 * *$ & $1257.94 * *$ & $92.01 * *$ & $53.16 * *$ & $218.10 * *$ & $32.69 * *$ \\
\hline $\begin{array}{l}\text { Irrigation within replication } \\
\text { (Pooled over year) }\end{array}$ & 7 & 42.13 & 0.09 & 0.01 & 0.15 & 0.09 & 0.19 \\
\hline $\begin{array}{l}\text { Irrigation within years within } \\
\text { replication (Pooled over year) }\end{array}$ & 15 & $166265.59 * *$ & $1128.45 * *$ & $841.61 * *$ & $631.36^{* *}$ & $1016.72 * *$ & $193.20 * *$ \\
\hline Lines $\times$ irrigation $\times$ year & 297 & 1.46 & 0.17 & 0.02 & 0.11 & 0.03 & 0.01 \\
\hline Error $\quad$ (Pooled over year) & 1485 & 37.18 & 12.68 & 0.30 & 0.37 & 2.20 & 3.19 \\
\hline
\end{tabular}

$*$, ** Significant at 5 and $1 \%$ level, respectively;

Leaf relative water content $(\%)=\mathrm{LRWC}$; Stomatal count $\left(\mathrm{mm}^{-2}\right)=\mathrm{SC}$; Canopy temperature before flowering $\left({ }^{\circ} \mathrm{C}\right)=\mathrm{CTF}$; Canopy temperature before maturity $\left({ }^{\circ} \mathrm{C}\right)=\mathrm{CTM}$; Chlorophyll content before flowering (SPAD units) = CCF; Chlorophyll content before maturity (SPAD units) = CCM 
Table.3 Analysis of variance for yield attributing traits and quality trait in inbred lines of maize (Zea mays L.) - Mean Sum of Squares (Pooled over years)

\begin{tabular}{|c|c|c|c|c|c|c|}
\hline Source of variation & d.f & EPP & KPR & 100GW & GYP & PC \\
\hline Replications & 1 & $0.81^{* *}$ & $719.21 * *$ & $103.83 * *$ & 71.80 & $0.43 * *$ \\
\hline Year & 1 & $5.04 * *$ & $14551.78 * *$ & $3034.52 * *$ & $5398729.00 * *$ & $57.74 * *$ \\
\hline Irrigations & 3 & $6.81 * *$ & $8502.75 * *$ & $1943.63 * *$ & $4120011.00^{* *}$ & $49.40 * *$ \\
\hline Lines & 99 & $1.13^{* *}$ & $133.28 * *$ & $64.67 * *$ & $70061.61 * *$ & $13.72 * *$ \\
\hline Lines $\times$ year & 99 & $0.93^{* *}$ & $1.10^{* *}$ & $1.35^{*}$ & $803.26^{* *}$ & $0.04 * *$ \\
\hline Line $\times$ irrigation & 297 & $1.01 *$ & $0.11 * *$ & $2.05 * *$ & $512.01 * *$ & $0.07 * *$ \\
\hline Irrigations $\times$ year & 3 & $0.37 * *$ & $1584.58 * *$ & $595.90 * *$ & $732946.10 * *$ & $3.94 * *$ \\
\hline Irrigation within replication (Pooled over year) & 7 & 0.01 & 0.69 & 0.10 & 0.68 & 0.01 \\
\hline $\begin{array}{l}\text { Irrigation within years within replication (Pooled } \\
\text { over year) }\end{array}$ & 15 & $1.82 * *$ & $3035.85 * *$ & $717.18 * *$ & $1330511.65^{* *}$ & $14.54 * *$ \\
\hline Lines $\times$ irrigation $\times$ year & 297 & 0.01 & 0.10 & 0.05 & $239.74 * *$ & 0.01 \\
\hline Error $\quad$ (Pooled over year) & 1485 & 0.02 & 4.72 & 0.65 & 204.75 & 0.01 \\
\hline
\end{tabular}

$*, * *$ Significant at 5 and $1 \%$ level, respectively

Ears plant $^{-1}=$ EPP; Kernels row ${ }^{-1}=$ KPR; 100-grain weight $(\mathrm{g})=100 \mathrm{GW}$; Grain yield plot $^{-1}(\mathrm{~g})=\mathrm{GYP}$; Protein content $(\%)=$ PC.

Table.4 Magnitude of variability for morphological, maturity, physiological, yield and quality traits in inbred lines of maize (Zea mays L.) over years

\begin{tabular}{|c|c|c|c|c|c|c|}
\hline \multirow[t]{2}{*}{ Source of variation } & \multicolumn{3}{|c|}{ Mean \pm SE } & \multicolumn{3}{|c|}{ Range } \\
\hline & Year-1 & Year-2 & Pooled & Year-1 & Year-2 & Pooled \\
\hline Days to $50 \%$ tasseling & $78.93 \pm 0.25$ & $78.46 \pm 0.30$ & $78.69 \pm 0.61$ & $68.12-83.62$ & $68.00-84.12$ & $68.06-83.87$ \\
\hline Days to $50 \%$ silking & $83.44 \pm 0.40$ & $82.97 \pm 0.45$ & $83.21 \pm 0.65$ & $71.12-88.87$ & $71.00-89.37$ & $71.06-89.12$ \\
\hline Anthesis-silking interval & $4.51 \pm 0.26$ & $4.51 \pm 0.26$ & $4.51 \pm 0.18$ & $3.00-5.50$ & $3.00-5.50$ & $3.00-5.50$ \\
\hline Days to maturity & $149.24 \pm 0.92$ & $149.24 \pm 0.50$ & $149.24 \pm 0.79$ & $136.50-155.37$ & $133.62-156.25$ & $136.50-155.81$ \\
\hline Plant height $(\mathbf{c m})$ & $157.64 \pm 1.35$ & $158.88 \pm 1.36$ & $158.26 \pm 0.93$ & $141.87-176.06$ & $143.06-177.25$ & $142.46-176.65$ \\
\hline Ear height $(\mathrm{cm})$ & $79.98 \pm 1.03$ & $80.73 \pm 0.68$ & $80.42 \pm 0.46$ & $71.63-90.20$ & $72.82-89.92$ & $72.52-89.62$ \\
\hline Leaf relative water content $(\%)$ & $93.81 \pm 3.15$ & $90.04 \pm 3.15$ & $91.92 \pm 2.1$ & $60.95-113.62$ & $57.24-109.80$ & $59.10-111.71$ \\
\hline Canopy temperature before flowering $\left({ }^{\circ} \mathrm{C}\right)$ & $32.45 \pm 0.15$ & $30.50 \pm 0.10$ & $31.47 \pm 0.19$ & $29.13-34.29$ & $27.27-32.33$ & $29.79-34.67$ \\
\hline Canopy temperature before maturity $\left({ }^{\circ} \mathrm{C}\right)$ & $20.37 \pm 0.12$ & $18.66 \pm 0.24$ & $19.52 \pm 0.21$ & $17.06-22.19$ & $14.91-20.48$ & $16.98-22.38$ \\
\hline Stomatal count $\left(/ \mathrm{mm}^{2}\right)$ & $80.06 \pm 1.84$ & $81.47 \pm 1.84$ & $80.76 \pm 1.25$ & $63.32-97.41$ & $64.75-98.84$ & $62.77-96.19$ \\
\hline Chlorophyll content before flowering (SPAD units) & $41.95 \pm 0.76$ & $40.47 \pm 0.76$ & $41.21 \pm 0.52$ & $34.12-55.99$ & $32.65-54.52$ & $31.97-52.96$ \\
\hline Chlorophyll content before maturity (SPAD units) & $11.97 \pm 0.92$ & $10.74 \pm 0.92$ & $11.36 \pm 0.63$ & $7.42-18.17$ & 6.13-17.07 & $6.41-16.72$ \\
\hline Ears plant $^{-1}$ & $1.08 \pm 0.07$ & $1.16 \pm 0.07$ & $1.12 \pm 0.05$ & $0.73-1.71$ & $0.79-1.84$ & $0.76-1.77$ \\
\hline Kernels row $^{-1}$ & $27.07 \pm 1.12$ & $24.40 \pm 1.12$ & $25.74 \pm 0.76$ & $22.58-32.29$ & $19.71-29.57$ & $21.14-30.93$ \\
\hline 100 grain weight $(\mathrm{g})$ & $21.95 \pm 0.41$ & $20.41 \pm 0.42$ & $21.18 \pm 0.28$ & $19.35-27.08$ & $17.74-25.62$ & $18.54-26.35$ \\
\hline Grain yield plot $^{-1}(\mathrm{~g})$ & $414.18 \pm 7.43$ & $411.07 \pm 7.37$ & $412.63 \pm 5.05$ & $357.43-635.62$ & $354.68-630.97$ & $356.05-633.30$ \\
\hline Protein content $(\%)$ & $7.67 \pm 0.02$ & $7.52 \pm 0.02$ & $7.60 \pm 0.02$ & $5.67-10.09$ & $5.47-9.96$ & $5.50-9.90$ \\
\hline
\end{tabular}


Table.5 Estimates of genotypic variance $\left(\sigma^{2} \mathrm{~g}\right)$, phenotypic variance $\left(\sigma^{2} \mathrm{p}\right), \mathrm{PCV}, \mathrm{GCV}$, heritability, genetic advance and genetic gain for morphological, maturity, physiological, yield and quality traits in inbred lines of maize (Zea mays L.) over years

\begin{tabular}{|c|c|c|c|c|c|c|c|c|c|c|c|c|}
\hline \multirow{2}{*}{ Traits } & \multicolumn{3}{|l|}{$\sigma^{2} g$} & \multicolumn{3}{|l|}{$\sigma^{2} p$} & \multicolumn{3}{|c|}{ GCV (\%) } & \multicolumn{3}{|c|}{$\operatorname{PCV}(\%)$} \\
\hline & Y1 & Y2 & Pool & Y1 & Y2 & Pool & Y1 & Y2 & Pool & Y1 & Y2 & Pool \\
\hline Days to $50 \%$ tasseling & 19.92 & 20.76 & 17.66 & 20.18 & 21.13 & 20.65 & 5.65 & 5.80 & 5.34 & 5.69 & 5.85 & 5.77 \\
\hline Days to $50 \%$ silking & 26.12 & 26.37 & 23.58 & 26.79 & 27.19 & 26.99 & 6.12 & 6.18 & 5.83 & 6.20 & 6.28 & 6.24 \\
\hline Days to maturity & 27.18 & 34.45 & 27.95 & 30.59 & 35.49 & 33.02 & 3.49 & 3.93 & 3.54 & 3.70 & 3.99 & 3.85 \\
\hline Anthesis-silking interval & 0.52 & 0.51 & 0.53 & 0.80 & 0.80 & 0.83 & 16.05 & 15.87 & 16.24 & 19.89 & 19.81 & 19.85 \\
\hline Plant height $(\mathrm{cm})$ & 69.05 & 70.72 & 70.37 & 76.40 & 78.18 & 77.29 & 5.27 & 5.29 & 5.30 & 5.54 & 5.56 & 5.55 \\
\hline Ear height $(\mathrm{cm})$ & 19.32 & 17.67 & 18.50 & 23.62 & 19.54 & 21.58 & 5.49 & 5.20 & 5.35 & 6.07 & 5.47 & 5.78 \\
\hline Leaf relative water content (\%) & 132.17 & 132.14 & 134.81 & 172.02 & 171.98 & 172.00 & 12.25 & 12.76 & 12.63 & 13.98 & 14.56 & 14.26 \\
\hline Canopy temperature before flowering $\left({ }^{\circ} \mathrm{C}\right)$ & 1.75 & 1.51 & 1.40 & 1.85 & 1.56 & 1.70 & 4.08 & 4.03 & 3.76 & 4.19 & 4.09 & 4.15 \\
\hline Canopy temperature before maturity $\left({ }^{\circ} \mathrm{C}\right)$ & 1.72 & 1.54 & 1.40 & 1.78 & 1.78 & 1.78 & 6.43 & 6.66 & 6.07 & 6.55 & 7.16 & 6.84 \\
\hline Stomatal count $\left(\mathrm{mm}^{-2}\right)$ & 79.03 & 78.62 & 79.73 & 92.62 & 92.21 & 92.41 & 11.10 & 10.88 & 11.05 & 12.02 & 11.78 & 11.90 \\
\hline $\begin{array}{l}\text { Chlorophyll content before flowering } \\
\text { (SPAD units) }\end{array}$ & 37.00 & 36.97 & 37.14 & 39.36 & 39.32 & 39.34 & 14.50 & 15.02 & 14.78 & 14.95 & 15.49 & 15.22 \\
\hline $\begin{array}{l}\text { Chlorophyll content before maturity } \\
\text { (SPAD units) }\end{array}$ & 8.22 & 8.35 & 8.51 & 11.64 & 11.77 & 11.71 & 23.94 & 26.88 & 25.68 & 28.49 & 31.92 & 30.11 \\
\hline Ears plant $^{-1}(\mathrm{EPP})$ & 0.06 & 0.06 & 0.07 & 0.08 & 0.09 & 0.09 & 24.01 & 22.42 & 23.38 & 27.26 & 25.99 & 26.61 \\
\hline Kernels row $^{-1}$ & 7.66 & 7.74 & 8.03 & 12.71 & 12.79 & 12.75 & 10.22 & 11.39 & 11.01 & 13.17 & 14.65 & 13.87 \\
\hline 100 grain weight $(\mathrm{g})$ & 3.92 & 3.98 & 4.00 & 4.63 & 4.69 & 4.66 & 9.02 & 9.77 & 9.44 & 9.80 & 10.60 & 10.19 \\
\hline 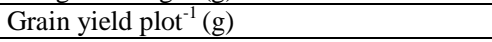 & 4382.03 & 4320.94 & 4366.05 & 4602.87 & 4538.75 & 4570.81 & 15.98 & 15.99 & 16.01 & 16.38 & 16.38 & 16.38 \\
\hline Protein content (\%) & 0.85 & 0.86 & 0.85 & 0.85 & 0.86 & 0.86 & 12.01 & 12.35 & 12.18 & 12.04 & 12.37 & 12.20 \\
\hline
\end{tabular}

\begin{tabular}{|c|c|c|c|c|c|c|}
\hline \multirow{2}{*}{ Traits } & \multicolumn{3}{|c|}{ Heritability $\left(\mathbf{h}^{2}\right)$} & \multicolumn{3}{|c|}{ Expected Genetic Gain (per cent of mean) } \\
\hline & Year-1 & Year-2 & Pool & Year-1 & Year-2 & Pool \\
\hline Days to $50 \%$ tasseling & 0.98 & 0.98 & 0.85 & 11.57 & 11.86 & 10.17 \\
\hline Days to $50 \%$ silking & 0.97 & 0.97 & 0.87 & 12.46 & 12.55 & 11.24 \\
\hline Days to maturity & 0.88 & 0.97 & 0.84 & 6.78 & 7.98 & 6.71 \\
\hline Anthesis-silking interval & 0.65 & 0.64 & 0.66 & 26.69 & 26.17 & 27.37 \\
\hline Plant height $(\mathrm{cm})$ & 0.90 & 0.90 & 0.91 & 10.32 & 10.37 & 10.41 \\
\hline Ear height $(\mathrm{cm})$ & 0.81 & 0.90 & 0.85 & 10.23 & 10.20 & 10.20 \\
\hline Leaf relative water content (\%) & 0.76 & 0.76 & 0.78 & 22.12 & 23.05 & 23.03 \\
\hline Canopy temperature before flowering $\left({ }^{\circ} \mathrm{C}\right)$ & 0.94 & 0.97 & 0.82 & 8.17 & 8.20 & 7.02 \\
\hline Canopy temperature before maturity $\left({ }^{\circ} \mathrm{C}\right)$ & 0.96 & 0.86 & 0.78 & 13.02 & 12.78 & 11.10 \\
\hline Stomatal count $\left(\mathrm{mm}^{-2}\right)$ & 0.85 & 0.85 & 0.86 & 21.12 & 20.70 & 21.15 \\
\hline Chlorophyll content before flowering (SPAD units) & 0.94 & 0.94 & 0.94 & 28.96 & 30.00 & 29.59 \\
\hline Chlorophyll content before maturity (SPAD units) & 0.70 & 0.70 & 0.72 & 41.44 & 46.64 & 45.10 \\
\hline Ears plant $^{-1}(\mathrm{EPP})$ & 0.77 & 0.74 & 0.77 & 43.56 & 39.85 & 42.33 \\
\hline Kernels row $^{-1}$ & 0.60 & 0.60 & 0.63 & 16.34 & 18.26 & 18.00 \\
\hline 100 grain weight $(\mathrm{g})$ & 0.84 & 0.84 & 0.85 & 17.11 & 18.56 & 18.01 \\
\hline Grain yield plot $^{-1}(\mathrm{~g})$ & 0.95 & 0.95 & 0.95 & 32.12 & 32.14 & 32.24 \\
\hline Protein content $(\%)$ & 0.99 & 0.99 & 0.99 & 24.71 & 25.40 & 25.04 \\
\hline
\end{tabular}

Contd... 
High estimates of heritability along with higher genetic advance are usually more useful than either of these parameters taken alone in predicting the resultant effect of selecting the best individuals (Johnson et al., 1955). Genetic advance being the function of heritability, selection intensity and phenotypic standard deviation indicates the magnitude of improvement in the desired direction that can be expressed in a particular character by selecting a certain proportion of population. Heritability (b.s.) was observed to be higher (> 60\%) for all the maturity, morphological, physiological, yield, quality traits in individual year, pooled over years analysis suggesting that selection for improvement of these characters would be effective through phenotypic selection. Similar results were reported by Rafiq et al., (2010), Olakojo and Olaoye (2011), Aminu and Izge (2012) and Azam et al., (2014). High heritability estimates is indicative to preponderance of additive gene action indicating character is less influenced by environmental effects. High estimates of broadsense heritability for most of the traits revealed that variations were transmitted to the progeny and indicated potential for developing high yielding varieties through selection of desirable plants in succeeding generations (Aminu and Izge, 2012). However, the selection for improvement of such characters may not be useful because broad sense heritability is based on total genetic variance which includes additive, dominant and epistatic variances. Thus, heritability values coupled with high genetic advance would be more reliable and useful on correlating selection criteria (Ram Reddy et al., 2012).

High heritability estimates with high genetic gain were observed in present set of lines for important drought related traits like anthesissilking interval, leaf relative water content, stomatal count, chlorophyll content, ears plant ${ }^{-1}$, grain yield plot ${ }^{-1}$ protein content over years and over different moisture management regimes. Similar results were reported by Mohammad et al., (2006), Ali et al., (2010) and Ram Reddy et al., (2012). High heritability estimates coupled with moderate genetic gain were observed in present set of lines for traits viz., days to $50 \%$ tasseling, days to $50 \%$ silking, plant height, ear height, canopy temperature before maturity, kernels row $^{-1}$ and 100 grain weight. High heritability with moderate genetic advance arises from dominance or epitasis as suggested by Paramasivan and Rangadwamy (1988). Similar results were reported by Jawaharlal et al., (2011), Anshuman et al., (2013) and Kumar et al., (2014). Therefore, for these traits hybridization followed by selection is expected to result in some promising recombinants for drought tolerance. Low estimates of genetic gain were revealed for days to maturity and canopy temperature before flowering. Results confirmed that phenotypic selection has a paramount significance for identification of elite lines as most of the characters exhibited variability due to genetic causes and thus, offers ample scope for improvement despite the fact that the two years could not be representative of random environment required to remove genotype $\mathrm{x}$ environment interaction, respectively. Maximum genetic gain was observed for chlorophyll content before maturity (45.10\%), anthesis-silking interval $(27.37 \%)$ and ear plant ${ }^{-1}(22.33 \%)$ indicating heritable nature of the characters for the materials under study.

\section{References}

Abuali, A.I., Abdelmula, A.A., Khalafalla, M.M., Idris, A.E. and Hamza, N.B. 2014. Assessment of genetic variability of inbred lines and their F1- hybrids of grain maize (Zea mays L.) under drought stress conditions. International Journal of Agronomy and Agricultural Research 5(2): 22-30.

Alake, C.O., Ojo, D.K., Oduwaye, O.A. and Adekoya, M.A. 2008. Genetic variability and correlation studies in yield and yield related characters of tropical maize (Zea mays L.). ASSET Series A 8(1): 14-27.

Ali, A.W., Hasan, K.A. and Samir, A. 2010. Genetic variances, heritability, correlation and path coefficient analysis in yellow 
maize crosses (Zea mays L.). Agriculture and Biology Journal of North America 1(4): 630-637.

Aminu, D. and Izge, A.U. 2012. Heritability and correlation estimates in maize (Zea mays L.) under drought conditions in northern Guinea and Sudan savannas of Nigeria. World Journal of Agricultural Sciences 8(6): 598-602.

Anonymous, 2013. Agro-Meterology, Division of Agronomy, SKUAST Kashmir, Shalimar.

Anonymous, 2014. Economic Survey 2013-14. Directorate of Economics and Statistics, Government of Jammu and Kashmir pp. 191-194.

Anshuman, V., Dixit, N.N., Dipika, Sharma, S.K. and Marker, S. 2013. Studies on heritability and genetic advance estimates in Maize genotypes. Bioscience Discovery 4(2): 165168.

Araus, J.L., Slafer, G.A., Reynolds, M.P. and Royo, C. 2002. Plant breeding and water relations in $\mathrm{C}_{3}$ cereals: what should we breed for? Annuals of Botany London 89: 925-940.

Azam, M.G., Sarker, U., Maniruzzam and Banik, B.R. 2014. Genetic variability of yield and its contributing characters on CIMMYT maize inbreds under drought stress. Bangladesh Journal of Agricultural Research 39(3): 419-426.

Azam, M.G., Sarker, U., Maniruzzam and Banik, B.R. 2014. Genetic variability of yield and its contributing characters on CIMMYT maize inbreds under drought stress. Bangladesh Journal of Agricultural Research 39(3): 419-426.

Banziger, M., Pixley, K.V., Vivek, B. and Zambezi, B.T. 2000. Characterization of elite maize germplasm grown in eastern and southern Africa: Results of the 1999 regional trials conducted by CIMMYT and the Maize and Wheat Improvement Research Network for SADC (MWIRNET). Zimbabwe pp. 1-44.

Barrs, H.D. and Weatherly, P.E. 1962. A reexamination of relative turgidity for estimating water deficits in leaves. Australian Journal of Biology Science 15: 413-428.

Bolanos, J. and Edmeades, G.O. 1993. Eight cycles of selection for drought tolerance in lowland tropical maize. II. Responses in reproductive behaviour. Field Crops Research 31: 253-268.

Briggs, F.N. and Knowels, P.F. 1967. Introduction to plant breeding. Reinhold Publishing Corporation. New York/London. Chapman, S.C., Crossa, J., Basfod, K.E. and kroonenberg, P.M. 1997. Genotype by environment effects and selection for drought tolerance in tropical maize. II. Three-mode pattern analysis. Euphytica, 95: 11-20.

Choudhary, A.K. and Chaudary, L.E. 2002. Genetic studies in some crosses of maize (Zea mays L.). Journal of Bisra Agricultural Research University 14: 87-90.

Critchley, W. and Klaus, S. 1991. A manual for the design and construction of water harvesting schemes for plant production.

DE and S, 2013. Economic Survey 2012-13. Directorate of Economics and Statistics Government of Jammu \& Kashmir, pp. 4-5.

Dubey, L., Prasanna, B.M., Hossain, F., Verma, D.K. and Ramesh, B. 2010. Phenotypic evaluation of a set of selected exotic maize inbred lines for drought stress tolerance. Indian Journal of Genetics 70(4): 355-362.

Hanson, C.H., Robinson, H.F. and Comstock, R.E. 1956. Biometrical studies of yield in segregating populations of Korean Lespedeza. Agronomy Journal 48(6): 268272.

Jawaharlal, J., Lakshmikantha, R.G. and Saikumar, R. 2011. Genetic variability and character association studies in maize. Agriculture Science Digest 31(3): 173-177.

Johnson, H.N., Robinson, H.F. and Comstock, R.E. 1955. Estimates of genetic and environmental variability in soybeans. Agronomy Journal 48: 314-318.

Joshi, P.K., Singh, N.P., Singh, N.N., Gerpacio, R.V. and Pingali, P.L. 2005. Maize in India: Production Systems, Constraints, and Research Priorities. CIMMYT, Mexico. D. F.

Kuchanur, P.H. 2010. Identification of drought tolerance maize (Zea mays L.) germplasm. Ph.D. Thesis submitted to University of Agricultural Sciences, Dharwad, Karnataka (India). 
Kumar, G.P., Reddy, V.N., Kumar, S.S. and Rao, P.V. 2014. Genetic variability, heritability and genetic advance studies in newly developed maize genotypes (Zea mays L.). International Journal of Pure and Applied Bioscience 2(1): 272-275.

Meseka, S.K., Menkir, A. and Ibrahim, A.E.S. 2008. Yield potential and yield stability of maize hybrids selected for drought tolerance. Journal of Applied Bioscience 3: 82-90.

Muhammad, A., Shabbir, M., Amer, H. and Muhammad, S. 2006. Evaluation of maize three way crosses through genetic variability, broad sense heritability, character association and path analysis. Journal of Agriculture Research 46(1): 3945.

Nepolean, T., Singh, I., Hossain, F., Pandey, N. and Gupta, H.S. 2012. Molecular characterization and assessment of genetic diversity of inbred lines showing variability for drought tolerance in maize. Journal of Plant Biochemistry and Biotechnology 22(1): 71-79.

Olakojo, S.A. and Olaoye, G. 2011. Correlation and heritability estimates of maize. African Journal of Plant Science 5(6): 365-369.

Paramasivan, K.S. and Rangadwamy, S.R. 1988. Study on variability and genetic advance in quantitative characters in rice. ORYZA 25(3): 301-306.

Praveenkumar, B. and Sridevi, O. 2014. Study of Genetic Variability in $S_{1}$ Maize (Zea mays L.) Inbred Lines Under Drought and Irrigated Conditions. Indian Journal of Animal Nutrition 7(16): 2267-2278.

Rafiq, C.M., Rafique, M. and Hussain, A. 2010. Studies on heritability, correlation and path analysis in maize (Zea mays L.). Journal of Agricultural Research 48: 1-35.
Ram Reddy, V., Seshagiri Rao, A. and Sudarshan, M.R. 2012. Heritability and character association among grain yield and its components in maize (Zea mays L.). Journal of Research. ANGRAU 40(2): 4549.

Saindass, Arora, P., Kumari, M. and Pal, D. 2001. Morphological traits determining drought tolerance in maize (Zea mays L.) Indian Journal of Agricultural Research 35(3): 190-193.

Salman, Saleem, Tahir, H.N. and Saleem U. 2011. Study of genetic variability in maize inbred lines under irrigated and drought conditions. International Journal of Agriculture and Applied Science 3(2): 8085.

Salman, Saleem, Tahir, H.N. and Saleem U. 2011. Study of genetic variability in maize inbred lines under irrigated and drought conditions. International Journal of Agriculture and Applied Science 3(2): 8085.

Umar, U.U., Ado, S.G., Aba, D.A. and Bugaje, S.M. 2015. Studies on genetic variability in maize (Zea mays L.) under stress and nonstress environmental conditions. International Journal of Agronomy and Agricultural Research 7(1): 70-77.

Williams, W. 1964. Genetical principles and plant breeding. Blackwell Scientific Publication. Oxford, London.

Zaidi, P.H. and Singh, N.N. 2005. Stresses on maize in tropics. Directorate of Maize Research, New Delhi, India.

Zaidi, P.H., Srinivasan, G., Cordova, H.S. and Sanchez, C. 2004. Gains from improvement for mid-season drought tolerance in tropical maize (Zea mays L.). Field Crops Research 89: 135-152.

\section{How to cite this article:}

Asima Gazal, F.A. Nehvi, Ajaz Ahmad Lone and Zahoor Ahmed Dar. 2017. Assessment of Genetic Variability of a Set of Maize Inbred Lines for Drought Tolerance under Temperate Conditions. Int.J.Curr.Microbiol.App.Sci. 6(12): 2380-2389. doi: https://doi.org/10.20546/ijcmas.2017.612.275 